\title{
Hybrid Wing Body Model Identification Using Forced- Oscillation Water Tunnel Data
}

\author{
Patrick C. Murphy ${ }^{1}$, Dan D. Vicroy ${ }^{2}$ \\ NASA Langley Research Center, Hampton, VA, 23681-2199 \\ Brian Kramer ${ }^{3}$ and Michael Kerho ${ }^{4}$ \\ Rolling Hills Research Corporation, El Segundo, CA, 90245-2822
}

\begin{abstract}
Static and dynamic testing of the NASA 0.7 percent scale Hybrid Wing Body (HWB) configuration was conducted in the Rolling Hills Research Corporation water tunnel to investigate aerodynamic behavior over a large range of angle-of-attack and to develop models that can predict aircraft response in nonlinear unsteady flight regimes. This paper reports primarily on the longitudinal axis results. Flow visualization tests were also performed. These tests provide additional static data and new dynamic data that complement tests conducted at NASA Langley 14- by 22-Foot Subsonic Tunnel. HWB was developed to support the NASA Environmentally Responsible Aviation Project goals of lower noise, emissions, and fuel burn. This study also supports the NASA Aviation Safety Program efforts to model and control advanced transport configurations in loss-of-control conditions.
\end{abstract}

\section{Nomenclature}

\begin{tabular}{|c|c|c|c|}
\hline$A_{j}, B_{j}$ & $=$ Fourier coefficients & $t$ & $=$ time, sec \\
\hline$a, b_{1}$ & $=$ deficiency function parameters & $V$ & $=$ velocity, fps \\
\hline$b$ & $=$ wing span, $\mathrm{ft}$ & $\alpha$ & $=$ angle-of-attack, rad or deg \\
\hline $\begin{array}{l}C_{N}, C_{Y} \\
C_{b} C\end{array}$ & $\begin{array}{l}=\text { normal and side force coefficients } \\
=\text { rolling and vawing-moment coefficients }\end{array}$ & $\alpha_{0}$ & $=\begin{array}{l}=\text { initial angle-of-attack in forced } \\
\text { oscillation experiments rad or deg }\end{array}$ \\
\hline $\begin{array}{l}C_{l}, C_{n} \\
C_{m}\end{array}$ & $\begin{array}{l}=\text { rolling and yawing-moment coefficients } \\
=\text { pitching-moment coefficients }\end{array}$ & $\beta$ & $\begin{aligned} & \text { oscillation experiments, rad or deg } \\
= & \text { sideslip angle, rad or deg }\end{aligned}$ \\
\hline $\bar{c}$ & $=$ mean aerodynamic chord, $\mathrm{ft}$ & $\eta$ & $=$ state variable \\
\hline$F_{a_{\alpha}}, F_{a_{\beta}}$ & $=$ deficiency functions & $\sigma$ & $=$ standard error \\
\hline$f$ & $=$ frequency, $\mathrm{Hz}$ & $\tau$ & $=$ dummy integration variable \\
\hline$k$ & $=$ reduced $f, \pi b f / V$, or $\pi \bar{c} f / V$ & $\tau_{1}$ & $=$ non-dimensional time constant, $\frac{1}{b}\left(\frac{2 V}{b}\right)$ \\
\hline $\begin{array}{l}m \\
N\end{array}$ & $\begin{array}{l}=\text { no. of harmonics in Fourier expansion } \\
=\text { number of data points }\end{array}$ & $\omega$ & $=$ angular frequency, $\mathrm{rad} / \mathrm{sec}$ \\
\hline$p, q, r$ & $=$ roll, pitch, and yaw rates, $\mathrm{rad} / \mathrm{sec}$ & $\bar{C}_{a_{\gamma}}$ & $=$ in-phase coefficients, $\gamma=\alpha$ or $\beta$ \\
\hline $\begin{array}{l}R^{2} \\
S\end{array}$ & $\begin{array}{l}=\text { coefficient of determination } \\
=\text { reference } a r e a, \mathrm{ft}^{2}\end{array}$ & $\bar{C}_{a_{\zeta}}$ & $=$ out-of-phase coefficients, $\zeta=p, q$, or $r$ \\
\hline $\mathrm{T}$ & $=$ dimensional time constant, sec & $S S_{E}, S S_{r}$ & $=$ residual and total sum of squares \\
\hline Subscripts & & Sup & ipts \\
\hline$A$ & $=$ amplitude & $\wedge$ & $=$ estimated value \\
\hline$a$ & $\begin{array}{l}=\text { aero forces and moments: } N, Y, l, m \text {, or } n \\
=\text { measured value }\end{array}$ & $\sim$ & $=$ mean value \\
\hline
\end{tabular}

\footnotetext{
${ }^{1}$ Senior Research Engineer, Dynamic Systems and Control Branch, Mail Stop 308, Associate Fellow.

${ }^{2}$ Senior Research Engineer, Flight Dynamics Branch, Mail Stop 308, Associate Fellow.

${ }^{3}$ President/CEO, Rolling Hills Research Corporation, 420 N Nash St, Senior Member.

${ }^{4}$ Chief Aerodynamicist, Rolling Hills Research Corporation, 420 N Nash St, Associate Fellow.
}

American Institute of Aeronautics and Astronautics 


\section{Introduction}

$\mathrm{T}$ HIS study contributes to development of NASA Hybrid Wing Body (HWB) aerodynamic models and to the investigation and development of mathematical models that can predict aircraft response in nonlinear unsteady flight regimes. A nonproprietary version of the HWB (HWB-N2A) is used in this study. Data from the tests provide additional information that complement tests conducted at NASA Langley $14 \times 22$ wind tunnel facility, for the NASA Environmentally Responsible Aviation (ERA) Project ${ }^{1}$. ERA Project goals are to develop advanced configurations with lower noise, emissions, and fuel burn ${ }^{2}$. This study also supports the NASA Aviation Safety Program, Vehicle Systems and Safety Technology Project, where project goals include model and control law development for advanced transport configurations in loss-of-control conditions ${ }^{3-4}$.

Application of a more general modeling approach to the HWB is presented in this paper. This approach allows for the inclusion of nonlinear behaviors and unsteady behaviors. The method takes advantage of indicial models that have been successfully applied to both transports and fighter aircraft over many years ${ }^{5-15}$. An advantage of the method is that the models retain conventional static and dynamic terms but replace conventional acceleration terms with indicial functions. This mathematical model structure allows unsteady aerodynamic behaviors to be represented under conditions that conventional series expansions of aerodynamic forces and moments cannot model. High fidelity aerodynamic models support development and application of simulation, analysis, and control design technology to enhance safety of flight of advanced transport configurations.

Static and dynamic testing of the NASA 0.7 percent scale Hybrid Wing Body (HWB) configuration was conducted in the Rolling Hills Research Corporation (RHRC) water tunnel to investigate aerodynamic behavior over a large flight regime and to develop models that can predict aircraft response in nonlinear unsteady flight regimes ${ }^{16}$. A very fine grid test matrix was used to fully characterize the aerodynamics. Flow visualization tests were also performed using dye ports along the leading edge of the wings and using a movable wand to investigate flows over the body. This paper presents static and dynamic test results, flow visualization graphics, and modeling analysis for the HWB. Data and analysis are primarily provided for the longitudinal axis in this paper. An additional study including comparisons of wind tunnel and water tunnel results is provided in Ref. 17.

\section{Water Tunnel Model}

The HWB model was designed to perform experiments in the RHRC research water tunnel, Model 2436. In order to maintain a wingspan equal to $75 \%$ of the tunnel width, a model scale of 0.007 to 1 was used. This resulted in a model wingspan of approximately 18 inches. The model is sting mounted, with the balance center located at the HWB moment reference center, which is located at the $38.7 \%$ mean aerodynamic chord. The balance is also located at buttline and waterline 0.0. Figure 1 shows three views of the general layout of the sting and balance inside the model. Reference parameters are: $S=70.263 \mathrm{in}^{2}, b=17.892 \mathrm{in}$, and $\bar{c}=7.398 \mathrm{in}$.

The model was constructed using several different materials. The main parts are shown in the CAD drawings as different colors. The main fuselage was made using a rigid plastic in a stereolithography (SLA) process. This was desirable because it allowed relatively easy and inexpensive routing of the dye tubes on the forebody. The nacelles, tails and fuselage cover plate were made of SLA material. These parts were painted and sealed with clearcoat to prevent water absorption. Because the wings and tails are so thin at this model scale, warpage was a major concern. For this reason, the wings were initially machined with a computer controlled milling machine out of a stiff nylon material and later rebuilt from solid aluminum and then hard anodized. The paint was removed from all of the other parts and they were primed with a marine barrier coat and then painted with marine gel coat.

The balance mounting plate, which was originally a flat surface built into the SLA fuselage, was not rigid enough and allowed the model angle-of-attack to change with relatively small pitching moment loads. To fix this, the surface was machined out of the model and a new stainless steel balance mounting plate was machined to fit in the pocket. Figure 1 shows the mounting plate in a salmon color at the front of the balance. The mount was epoxied into place in the body, which eliminated the deflections under load. The basic water tunnel model performed very well for many hours of continuous use.

\section{Measurements}

A series of static tests ${ }^{1}$ on the HWB were completed in 2012 at the NASA Langley 14-by 22-Foot Subsonic Tunnel. The water tunnel was used in 2013 to obtain some early dynamic data including some comparison static data and flow visualization data. Measurements were made for all three axes with an emphasis on the pitch-axis forced oscillation tests. Tests in the water tunnel were conducted at a speed of $11 \mathrm{in} / \mathrm{sec}$, corresponding to a Reynolds Number of $5.8 \times 10^{4}$ based on mean aerodynamic chord. The steady data were obtained for cruise and droop-wing configurations over a wide range of angle-of-attack from $-10^{\circ}$ to $30^{\circ}$, and sideslip angles from $-10^{\circ}$ to 
$10^{\circ}$. The dynamic tests included one degree-of-freedom forced oscillation around the pitch, roll, and yaw axes at different nominal values of the angle-of-attack, for different frequencies and amplitudes. Commanded oscillations were in the form of single sinusoids, Schroeder sweeps, and ramp-and-hold inputs. Dynamic test results presented in this paper are for the cruise configuration under sinusoidal forced oscillation.

The longitudinal oscillatory data were taken for the cruise configuration, at ten nominal values of angle-of-attack in the range $0^{\circ}$ to $20^{\circ}$, three amplitudes of $2.5^{\circ}, 5^{\circ}$, and $10^{\circ}$, and eight non-dimensional frequencies ranging from 0.05 to 0.30 or dimensional frequencies in the range of $0.0240 \mathrm{~Hz}$ to $0.1437 \mathrm{~Hz}$. During the test runs, data were obtained for approximately 30 oscillation cycles. Oscillatory data were sampled at $15 \mathrm{~Hz}$ with $5 \mathrm{~Hz}$ analog and $2 \mathrm{~Hz}$ digital filters. The resulting data were further filtered with a $0.6 \mathrm{~Hz}$ low pass digital filter to remove unwanted frequency content. The filter was run in both directions to ensure no phase error was added to the data. Initial cycles were discarded to avoid any startup transients in the data and typically a minimum of 10 repeated cycles were used for data analysis to ameliorate the effects of any measurement noise. A wide range of filters were tested to ensure the final results were not impacted by the filter cut-off frequency. Lateral-directional oscillatory tests were performed in a similar manner at six nominal values of angle-of-attack in the range $2^{\circ}$ to $22^{\circ}$, two amplitudes of $5^{\circ}$, and $20^{\circ}$, and nine non-dimensional frequencies ranging from 0.05 to 0.35 or dimensional frequencies in the range of $0.0098 \mathrm{~Hz}$ to $0.0685 \mathrm{~Hz}$.

Static data for normal force and pitching moment coefficients, over a $\pm 10^{\circ}$ range of sideslip, are shown in Figs. 2 and 3. Normal force was not affected and fairly limited variation occurs in the pitching moment coefficient with changes in sideslip. Figures 4 and 5 show the same coefficients compared with wind tunnel measurements for the $\beta$ $=0^{\circ}$ alpha sweep. In comparison to wind tunnel results, the normal force compares fairly well. The slope compares well but the absolute levels are low. The distinct break in the wind tunnel results at stall are also not present in the water tunnel results. Due to the very low Reynolds number, the boundary layers are all laminar. The laminar boundary layers, coupled with the high aspect ratio design, mean that the wings will under perform, producing less lift than the higher Reynolds number wind tunnel data. The pitching moment results present levels that are offset from the wind tunnel data with the water tunnel data being more positive as the flow over the wings separates prematurely.

Sample flow visualization results are shown for $0^{\circ}, 10^{\circ}$, and $15^{\circ}$ angle-of-attack at $0^{\circ}$ sideslip. More cases and details can be obtained in the RHRC final report ${ }^{16}$. Flow visualization obtained with dye ports is shown in Fig. 6 and with a movable wand in Figs. 7 and 8. These graphics show a well behaved flow field and normal stalled flow formations at $10^{\circ}$ and $15^{\circ}$ angle-of-attack where more turbulent flow appears near the rear wing root.

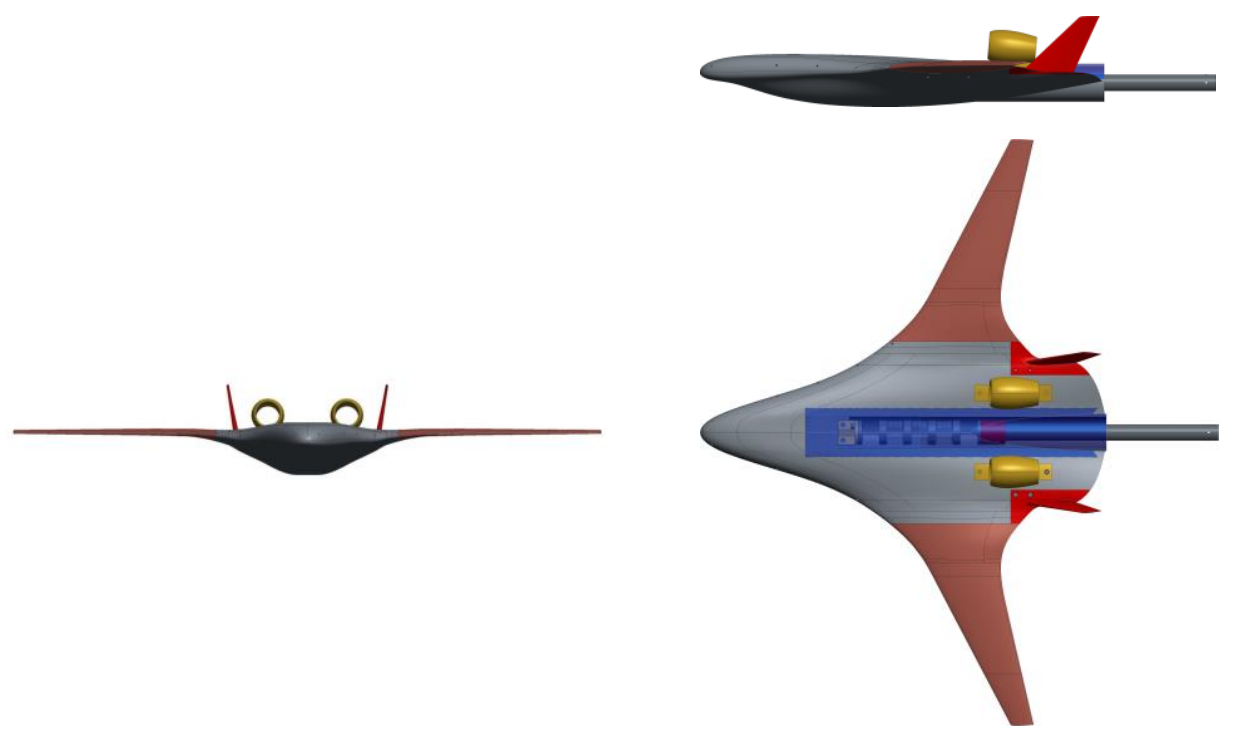

Figure 1. Model of the NASA Hybrid Wing Body with sting built for static and dynamic testing in Rolling Hills Research Corporation water tunnel. 


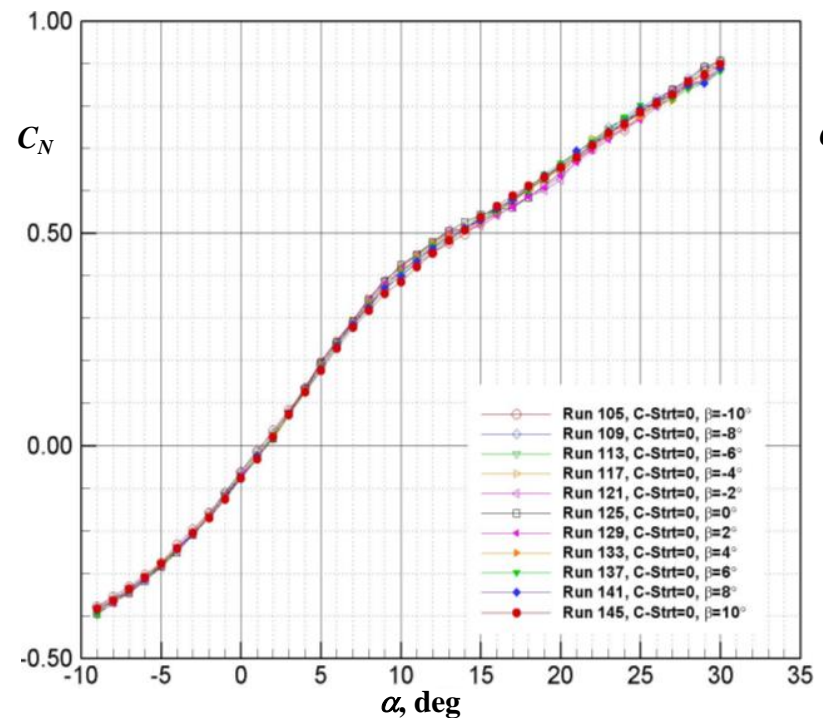

Figure 2. Static normal force coefficient.

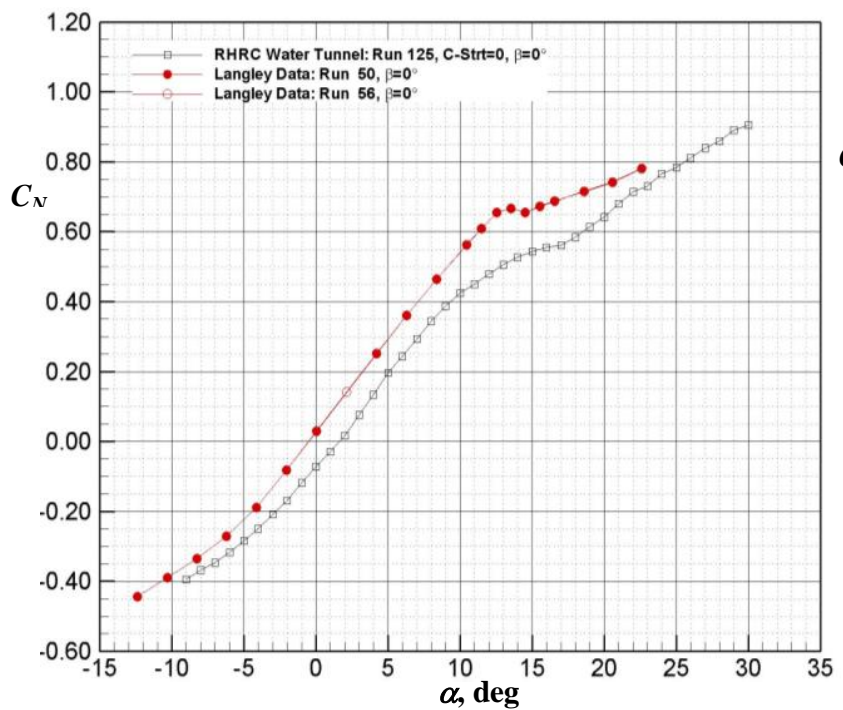

Figure 4. Comparison of water and wind tunnel normal force coefficient, $\beta=0^{\circ}$.

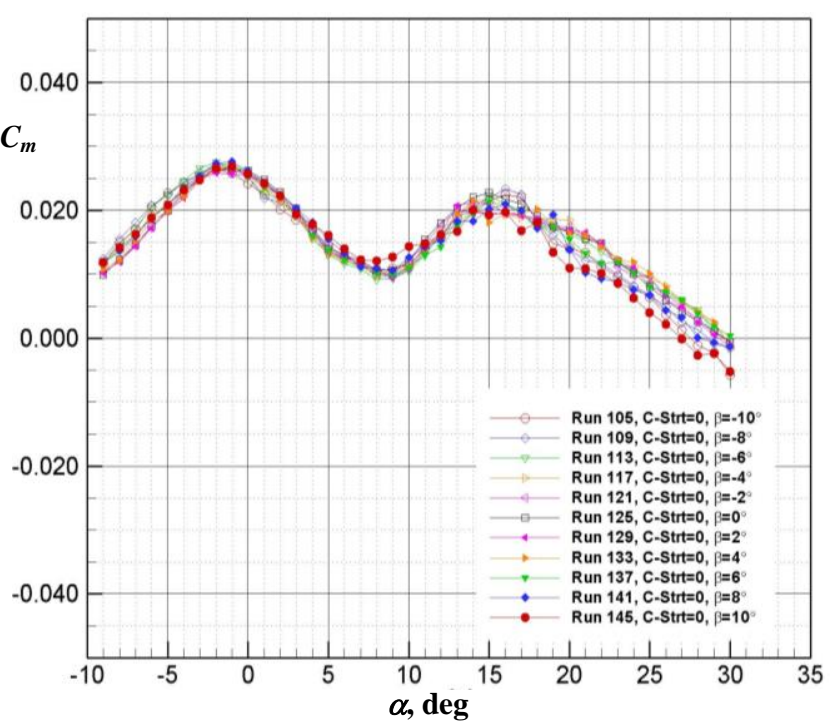

Figure 3. Static pitching moment coefficient.

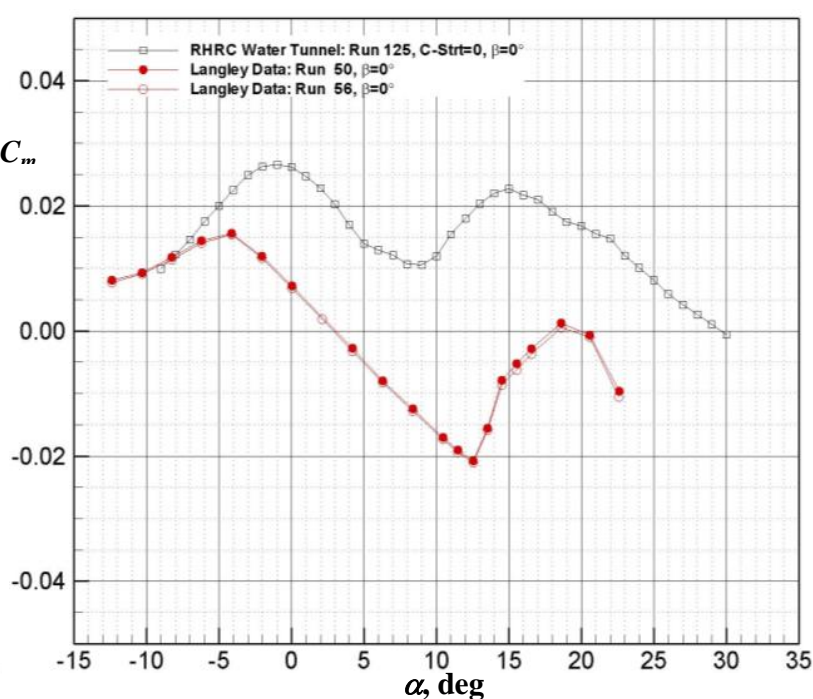

Figure 5. Comparison of water and wind tunnel pitching moment coefficient, $\beta=0^{\circ}$.

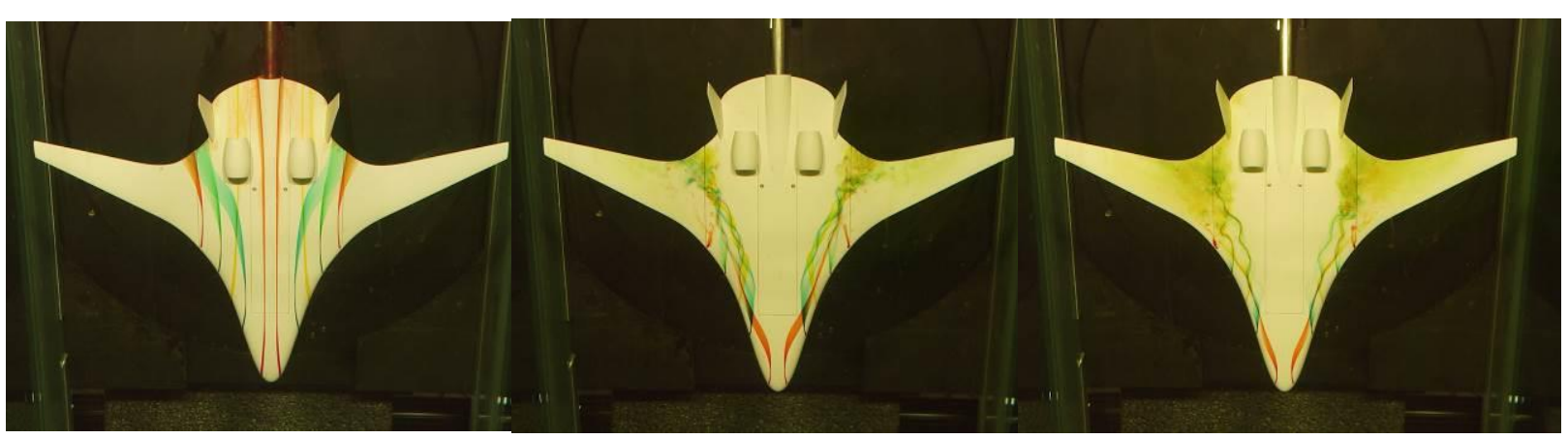

Figure 6. Flow visualization at $0^{\circ}, 10^{\circ}$, and $15^{\circ}$ angle-of-attack and $0^{\circ}$ sideslip.

4

American Institute of Aeronautics and Astronautics 


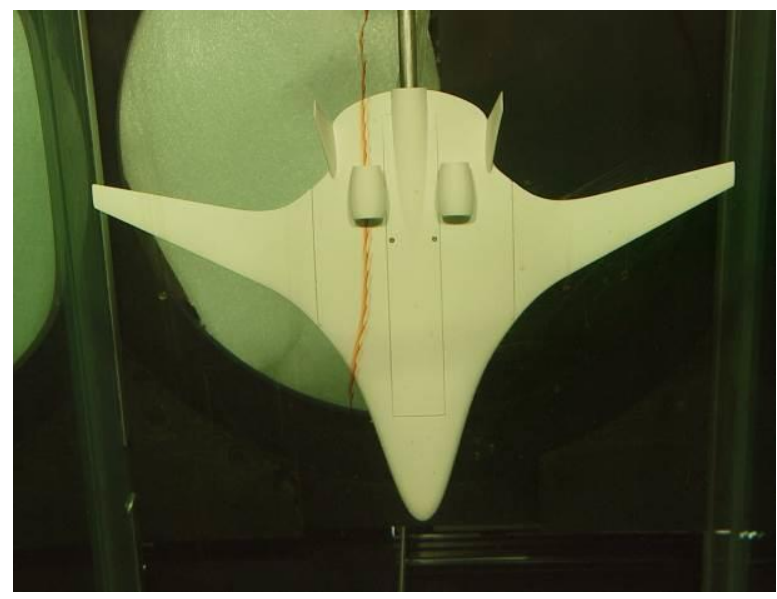

Figure 7. Flow visualization with dye wand over center body at $10^{\circ}$ angle-of-attack and $0^{\circ}$ sideslip.

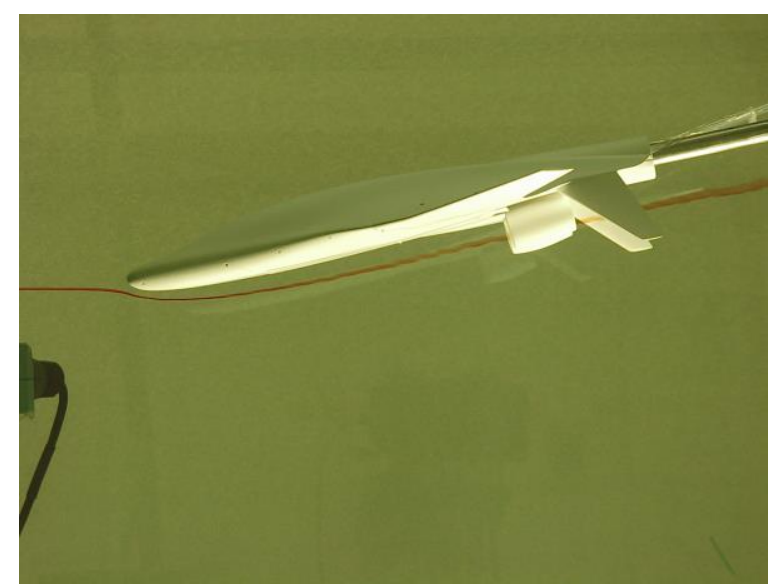

Figure 8. Flow visualization with dye wand off body at $10^{\circ}$ angle-of-attack and $0^{\circ}$ sideslip.

\section{Model Structure with Unsteady Aerodynamics}

Model structures for the aerodynamic coefficients, $C_{N}, C_{m}, C_{Y}, C_{n}$, and $C_{l}$, represented by $C_{a}$, where subscript $a=N, m, Y, n$, or $l$, were developed from a general form of the indicial model equations presented in Refs. 5-7. A more general set of equations was developed for the wing-tail combinations allowing the wing and tail contributions to be treated separately in Refs 6 and 8. For the HWB configuration, however, a simpler model structure is used that allows the same form for both longitudinal and lateral-directional axes. In this case each lateral-directional coefficient can be written as

$$
C_{a}(t)=C_{a}(0)+\int_{0}^{t} C_{a_{\beta}}(t-\tau) \dot{\beta}(\tau) d \tau+\frac{b}{2 V} \int_{0}^{t} C_{a_{p}}(t-\tau) \dot{p}(\tau) d \tau+\frac{b}{2 V} \int_{0}^{t} C_{a_{r}}(t-\tau) \dot{r}(\tau) d \tau
$$

and the longitudinal coefficients can be written as

$$
C_{a}(t)=C_{a}(0)+\int_{0}^{t} C_{a_{\alpha}}(t-\tau) \dot{\alpha}(\tau) d \tau+\frac{\bar{c}}{2 V} \int_{0}^{t} C_{a_{q}}(t-\tau) \dot{q}(\tau) d \tau
$$

In Eqs. (1a) and (1b) coefficients $C_{a_{\alpha}}(t), C_{a_{q}}(t), C_{a_{\beta}}(t), C_{a_{p}}(t)$, and $C_{a_{r}}(t)$ are the indicial functions and $C_{a}(0)$ is the initial value of $C_{a}$. From Ref. 7, two assumptions are adopted to simplify the model: (a) the effect of angular accelerations $\dot{q}, \dot{p}$, and $\dot{r}$ on any coefficient can be neglected and (b) the indicial functions in Eq. (1) can be expressed for the lateral-directional case as

$$
C_{a_{\beta}}(t)=C_{a_{\beta}}(\infty)-F_{a_{\beta}}(t)
$$

and for the longitudinal case as

$$
C_{a_{\alpha}}(t)=C_{a_{\alpha}}(\infty)-F_{a_{\alpha}}(t)
$$

where $F_{a_{\alpha}}(t)$ and $F_{a_{\beta}}(t)$ are the deficiency functions and $C_{a_{\alpha}}(\infty)$ and $C_{a_{\beta}}(\infty)$ are the rates of change $C_{a}$ with $\alpha$ and $\beta$, respectively, evaluated in steady flow conditions. 
The simplified model, which takes into account changes with respect to steady state, has the lateral-directional form

$$
C_{a}(t)=C_{a_{\beta}}(\infty) \beta(t)+\frac{b}{2 V} C_{a_{p}}(\infty) p(t)+\frac{b}{2 V} C_{a_{r}}(\infty) r(t)-\int_{0}^{t} F_{a_{\beta}}(t-\tau) \dot{\beta}(\tau) d \tau
$$

and the longitudinal form

$$
C_{a}(t)=C_{a_{\alpha}}(\infty) \alpha(t)+\frac{\bar{c}}{2 V} C_{a_{q}}(\infty) q(t)-\int_{0}^{t} F_{a_{\alpha}}(t-\tau) \dot{\alpha}(\tau) d \tau
$$

To obtain a model appropriate for identification and with a limited number of parameters, the deficiency function is assumed to be a simple exponential function ${ }^{7}$

$$
F_{a_{\gamma}}=a e^{-b_{1} t}
$$

where $\gamma$ can be $\alpha$ or $\beta$ depending on axis being modeled. Models appropriate for an aircraft undergoing one degreeof-freedom forced oscillation in roll or yaw can be obtained using Eqs. (3) and (4). Considering one degree-offreedom rolling motion in the tunnel

$$
C_{a}(t)=C_{a}[\phi(t), p(t)]
$$

where sideslip angle is related to the roll angle by the equation

$$
\beta(t)=\sin ^{-1}(\sin \alpha(t) \sin \phi(t))
$$

Combining Eqs. (3-6), the aerodynamic models can be formulated as

$$
C_{a}(t)=C_{a_{\beta}}(\infty) \beta(t)+\frac{b}{2 V} C_{a_{p}}(\infty) p(t)-a \int_{0}^{t} e^{-b_{1}(t-\tau)} \dot{\beta}(\tau) d \tau
$$

By introducing

$$
\eta(t)=\int_{0}^{t} e^{-b_{1}(t-\tau)} \dot{\beta}(\tau) d \tau
$$

and applying the Leibniz integral rule, the state space form of Eq. (7) can be written as

$$
\begin{gathered}
\dot{\eta}(t)=-b_{1} \eta(t)+\dot{\beta}(t) \\
C_{a}(t)=C_{a_{\beta}}(\infty) \beta(t)+\frac{b}{2 V} C_{a_{p}}(\infty) p(t)-a \eta(t)
\end{gathered}
$$

From Eq. (7), a steady response can be obtained ${ }^{7}$ as

$$
C_{a}(t)=\bar{C}_{a \beta} \phi_{A} \sin (\omega t)+\bar{C}_{a_{p}} \phi_{A} k \cos (\omega t)
$$


Where $\phi_{A}$ is the amplitude of roll oscillation, $k$ is reduced frequency, and $\bar{C}_{a_{\beta}}$ and $\bar{C}_{a_{p}}$ are the in-phase and out-ofphase components, respectively. These components are related to the model parameters (aerodynamic derivatives) by the equations ${ }^{7}$

$$
\begin{gathered}
\bar{C}_{a_{\beta}}=C_{a_{\beta}}(\infty) \sin \alpha_{0}-a \frac{\tau_{1}^{2} k^{2}}{1+\tau_{1}^{2} k^{2}} \sin \alpha_{0} \\
\bar{C}_{a_{p}}=C_{a_{p}}(\infty)-a \frac{\tau_{1}}{1+\tau_{1}^{2} k^{2}} \sin \alpha_{0}
\end{gathered}
$$

For one degree-of-freedom yaw oscillations in the tunnel

$$
C_{a}(t)=C_{a}[\psi(t), r(t)]
$$

where yaw and sideslip angles are related by the equation

$$
\beta(t)=\sin ^{-1}(-\cos \alpha(t) \sin \psi(t))
$$

The state space equations can be written as

$$
\begin{gathered}
\dot{\eta}(t)=-b_{1} \eta(t)+\dot{\beta}(t) \\
C_{a}(t)=C_{a_{\beta}}(\infty) \beta(t)+\frac{b}{2 V} C_{a_{r}}(\infty) r(t)-a \eta(t)
\end{gathered}
$$

and the corresponding in-phase and out-of-phase components ${ }^{7}$ are expressed as

$$
\begin{gathered}
\bar{C}_{a_{\beta}}=C_{a_{\beta}}(\infty) \cos \alpha_{0}-a \frac{\tau_{1}^{2} k^{2}}{1+\tau_{1}^{2} k^{2}} \cos \alpha_{0} \\
\bar{C}_{a_{r}}=C_{a_{r}}(\infty)+a \frac{\tau_{1}}{1+\tau_{1}^{2} k^{2}} \cos \alpha_{0}
\end{gathered}
$$

For the longitudinal axis a similar derivation results in the following expressions. For one degree-of-freedom pitch oscillations in the tunnel

$$
C_{a}(t)=C_{a}[\theta(t), q(t)]
$$

where pitch and angle-of-attack are constrained by the physical test arrangement and thus related by the equality (for upright oscillations)

$$
\alpha(t)=\theta(t)
$$

The state space equations can be written as

$$
\dot{\eta}(t)=-b_{1} \eta(t)+\dot{\alpha}(t)
$$




$$
C_{a}(t)=C_{a_{\alpha}}(\infty) \alpha(t)+\frac{\bar{c}}{2 V} C_{a_{q}}(\infty) q(t)-a \eta(t)
$$

and the corresponding in-phase and out-of-phase components ${ }^{7}$ are expressed as

$$
\begin{aligned}
& \bar{C}_{a_{\alpha}}=C_{a_{\alpha}}(\infty)-a \frac{\tau_{1}^{2} k^{2}}{1+\tau_{1}^{2} k^{2}} \\
& \bar{C}_{a_{q}}=C_{a_{q}}(\infty)-a \frac{\tau_{1}}{1+\tau_{1}^{2} k^{2}}
\end{aligned}
$$

\section{Model Identification}

Two main steps in system identification are model structure determination and parameter estimation. Model structures presented in the last section have a form that allows parameters to be estimated using an output error method; however, some additional details on model order and degree of nonlinearity are required before parameter estimation is performed. Harmonic analysis is useful for that purpose by characterizing where, with respect to angleof-attack and sideslip, unsteady behaviors occur and determining to what degree a nonlinear formulation is required. Harmonic analysis along with the coefficient of determination, $R^{2}$, can reveal these characteristics. $R^{2}$ is sensitive to noise so the analysis and interpretation of the coefficient can be made more difficult with low signal/noise data. Harmonic analysis is briefly described next; however, some discussion of the best practices for harmonic analysis and dealing with the effects of noise are presented more fully in Ref. 14.

\section{A. Harmonic Analysis}

A method of harmonic analysis ${ }^{11}$ was applied to measured aerodynamic coefficients. A mathematical model for these coefficients is

$$
C_{a}(t)=A_{0}+\sum_{j=1}^{m} A_{j} \cos (j \omega t)+\sum_{j=1}^{m} B_{j} \sin (j \omega t) \quad a=l, n \text { or } \mathrm{Y}
$$

where $A_{0}, A_{j}$, and $B_{j}$ are the Fourier coefficients. The analysis provides estimates of these coefficients, their standard errors, and the coefficient of determination, $R^{2}$. For the model with linear aerodynamics and $A_{0}=0$, the aerodynamic in-phase and out-of-phase components can be expressed in terms of the coefficients $A_{1}$ and $B_{1}$. For the roll oscillation case the expressions are

$$
\begin{gathered}
\bar{C}_{a_{\beta}}=\frac{B_{1}}{\phi_{A}} \\
\bar{C}_{a_{p}}=\frac{A_{1}}{k \phi_{A}}
\end{gathered}
$$

For the yaw oscillation case the expressions are

$$
\begin{gathered}
\bar{C}_{a_{\beta}}=\frac{B_{1}}{\psi_{A}} \\
\bar{C}_{a_{r}}=\frac{A_{1}}{k \psi_{A}}
\end{gathered}
$$


where $\beta$ is related to $\phi$ and $\psi$ by Eqs. (6) and (15). For pitch oscillation the expressions are

$$
\begin{gathered}
\bar{C}_{a_{\alpha}}=\frac{B_{1}}{\alpha_{A}} \\
\bar{C}_{a_{q}}=\frac{A_{1}}{k \alpha_{A}}
\end{gathered}
$$

The coefficient of determination, $R^{2}$, indicates the fraction of the variation in the measured data explained by the model and is defined as

$$
R^{2}=1-S S_{E} / \mathrm{SS}_{r} \quad 0<R^{2}<1
$$

where

$$
S S_{E}=\sum_{i=1}^{N}\left[C_{a_{E}}(i)-\hat{C}_{a}(i)\right]^{2}
$$

is the residual sum of squares and

$$
S S_{r}=\sum_{i=1}^{N}\left[C_{a_{E}}(i)-\tilde{C}_{a}(i)\right]^{2}
$$

is the total sum of squares. $C_{a_{E}}(i), \hat{C}_{a}(i)$, and $\tilde{C}_{a}(i)$ are the measured, estimated, and mean values, respectively.

\section{B. Parameter Estimation}

Careful input design with a wide range of amplitudes and frequencies will provide sufficient information content for complete model identification. Parameter estimation for this study was accomplished using a variation on an output-error method ${ }^{9,15,18}$ in the time domain. In this approach the model equations are given by the state-space equations, noted in section IV, corresponding to the axis of interest. For the examples in this study, the longitudinal axis Eqs. (22) and (23) were applied. Measured time histories of the inputs, $\alpha$ or $\beta$, and outputs, $C a$, for each of the frequencies tested are combined or "stacked" to ensure all the frequency content is included in the time domain estimation process. Equation integrations must be performed separately for each oscillation test but a single cost function is used to capture the overall model effectiveness. Application of the general output-error method for aircraft is explained and relevant software is provided in Ref. 19.

\section{Modeling Results and Discussion}

Harmonic analysis was performed to test the aerodynamic model frequency response and to test the degree of nonlinearity for the HWB configuration. Sample results of harmonic analysis are shown in Fig. 9 for pitch axis oscillations. The values were obtained using the first harmonic and Eqs (31) and (32). The figure presents normal force and pitching moment out-of-phase and in-phase components as a function of angle-of-attack and frequency. The results indicate very little frequency spread (unsteady behavior) over the entire range of angle-of-attack for both normal-force and pitching-moment out-of-phase components and a limited spread for the normal-force in-phase component. The scales have been adjusted to magnify the very limited variation in magnitude at the different frequencies.

Figure 10 shows the out-of-phase damping term for the HWB as a function of frequency, at four angles-ofattack, and a representative amplitude, $\alpha_{A}=10^{\circ}$. The lack of frequency dependence indicates that no unsteady behaviors occur for the conditions tested. In this case, without any frequency dependence present, investigators would normally test at lower frequencies to check for the characteristic "knee" in the curve of damping vs frequency. However, the extremely small values of $\bar{C}_{m_{q}}$ at high frequency implies that this particular aircraft has 
characteristically little damping in pitch and likely very limited unsteady responses even for lower frequencies. Future tests will verify this assumption regarding responses at lower frequencies. For a relative comparison, a conventional transport tested without the tail (only body-wing configuration) would present damping coefficients (out-of-phase coefficients) one to two orders of magnitude greater than presented in Fig. 9b, i.e., magnitudes in the range of 2 to 20. The scales in Fig. 10 are adjusted to a more representative magnitude of wing damping to demonstrate the frequency independence.

Values of $R^{2}$ are consistently above 0.90 for the normal force coefficients indicating that a conventional linear aerodynamic model should be adequate for that coefficient. $R^{2}$ for the pitching moment coefficients are very low inidicating that a higher order model (nonlinear model) is required. Higher-order harmonics were found to improve the model fit. A $2^{\text {nd }}$-order harmonic model improved $\bar{C}_{m_{q}}$ model fit such that $R^{2}$ implied $70 \%$ of the variation was explained by the model and a $3^{\text {rd }}$ order model improved model fit such that $R^{2}$ implied $81 \%$ of the variation was explained. Figure 11 shows the progressive improvement in $R^{2}$ for the three different harmonic orders considered. A cubic nonlinearity is expected since the forced oscillation about $\alpha_{0}=10^{\circ}$ with amplitude $\alpha_{A}=10^{\circ}$ covers a range of the underlying static curve that is cubic in nature.

The cubic characteristic found from harmonic analysis establishes a likely model structure for use during parameter estimation. Since the unsteady terms have been eliminated, the only source of nonlinearity must come from either static or steady-flow rotary dynamic terms. One option is to use stepwise regression, as used in Ref. 8, to select the best model structure. Another option, for low-order polynomials with only a few terms, is a trial-and-error approach that may be as fast and effective. The static model is available from measurements; so, estimating the static terms can be avoided by using the measured model directly. In that case, the identification problem can be simplified to estimating the best polynomial order for the damping terms. A progressive test of higher order terms can quickly reveal the model order required for the steady-flow damping. This approach resulted in a $3^{\text {rd }}$ order polynomial expression for the damping term as a function of angle-of-attack. Figure 12 shows measurements of the static pitching moment curve (blue) and forced-oscillation pitching-moment measurements (green) at each of the 8 test frequencies. Each of the responses, shown as $\delta C_{m}$ versus $\delta \alpha$, have the nominal estimated static values subtracted out and represent displacements from the nominal test condition at $\alpha_{0}=10^{\circ}$ with oscillation amplitude $\alpha_{A}=10^{\circ}$. Estimated nominal static values from dynamic data are slightly different than the measured static values, hence the measured static curve does not pass exactly through 0 for $\delta \alpha=0$. In addition the model-predicted responses (red) are shown where the steady-flow damping term has been estimated using a cubic polynomial representation. The steady-flow, nonlinear damping term in pitch was estimated as

$$
C_{m_{q}}(\infty ; \alpha)=-0.7155-3.7165 \alpha+10.5296 \alpha^{2}+111.1288 \alpha^{3}
$$

A nonlinear polynomial for the static curve, within the range of oscillation, was also estimated for this case as

$$
C_{m}(\infty ; \alpha)=-0.0060+0.0136 \alpha+0.3956 \alpha^{2}-0.6384 \alpha^{3}
$$

Overall, these models represent a very good fit to the measured responses at each frequency. Responses show the expected behavior of the dynamic response moving closer to the static curve as frequency is reduced. The estimation process is done including all frequencies simultaneously in order to ensure the best overall representation for time history responses. This was accomplished by "stacking" responses of each frequency in a modified output-error method discussed previously in section V.B. In this case, the $R^{2}$ achieved by these models was 0.904 or in other words $90 \%$ of the variation was explained by the model over all the frequencies. Explaining $90 \%$ of the variation is an excellent result in any dynamic test environment. Poor measurement quality (signal/noise ratio) or degree of nonlinearity can limit the achievable $R^{2}$ values. Polynomial coefficients and their standard errors are given in the table below. Mean values at the nominal or mean oscillation test point are subtracted from the measurements during output-error estimation so the first term, $A_{0}$, is not estimated for the static term $C_{m}(\infty ; \alpha)$ polynomial. The best estimate of the static polynomial term, $A_{0}$, is the static value of the pitching moment at $\alpha_{0}$. 
Table: Polynomial coefficients and their standard errors for $C_{m}(\infty ; \alpha)$ and $C_{m_{q}}(\infty ; \alpha)$ third-order models.

\begin{tabular}{|c|l|l|l|l|l|}
\hline Coefficients & \multicolumn{2}{|c|}{$A_{0}(\hat{\sigma})$} & \multicolumn{2}{|c|}{$A_{1}(\hat{\sigma})$} & \multicolumn{2}{|c|}{$A_{2}(\hat{\sigma})$} & \multicolumn{2}{c|}{$A_{3}(\hat{\sigma})$} \\
\hline$C_{m}(\infty ; \alpha)$ & N/A & $0.0136 \quad(0.0007)$ & $0.3956 \quad(0.0024)$ & $-0.638 \quad(0.028)$ \\
\hline$C_{m_{q}}(\infty ; \alpha)$ & $-0.7155(0.0022)$ & $-3.717 \quad(0.041)$ & $10.53 \quad(0.21)$ & $111.13 \quad(2.38)$ \\
\hline
\end{tabular}
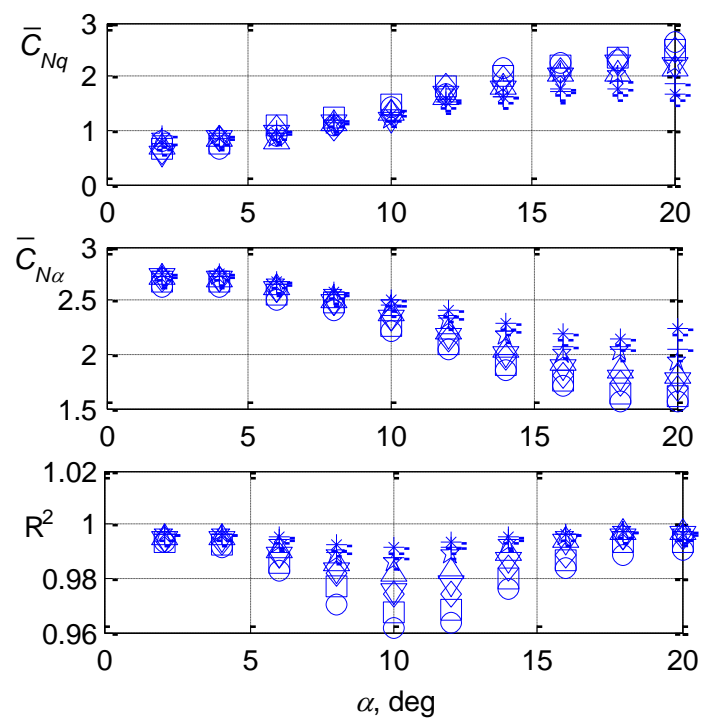

Figure 9a. $1^{\text {st }}$-order harmonic analysis for Normal force coefficient, $\alpha_{A}=10^{\circ}$.

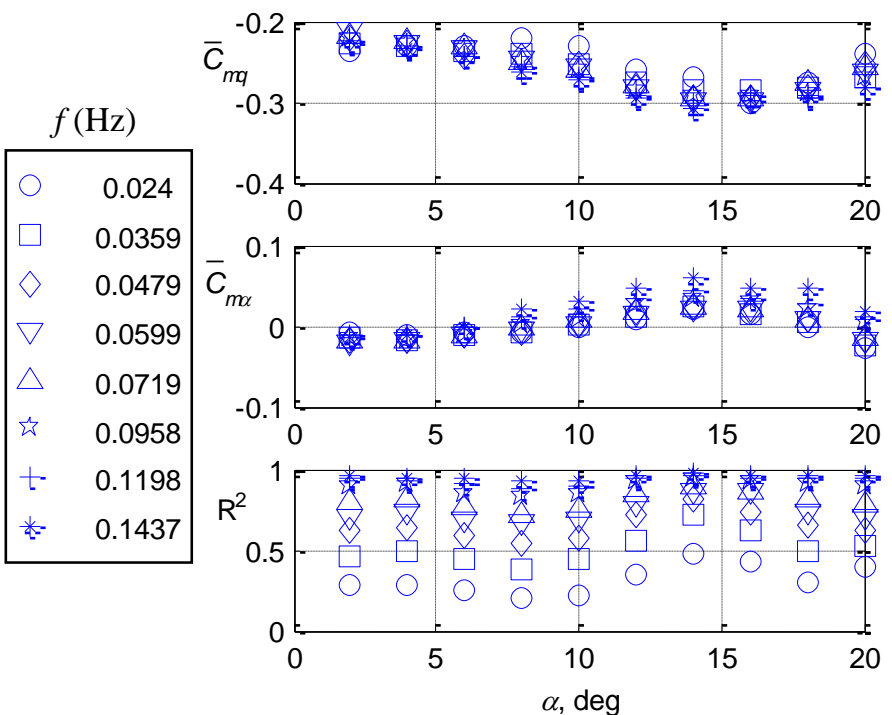

Figure 9b. $1^{\text {st }}$-order harmonic analysis for pitching moment coefficient, $\alpha_{A}=10^{\circ}$.

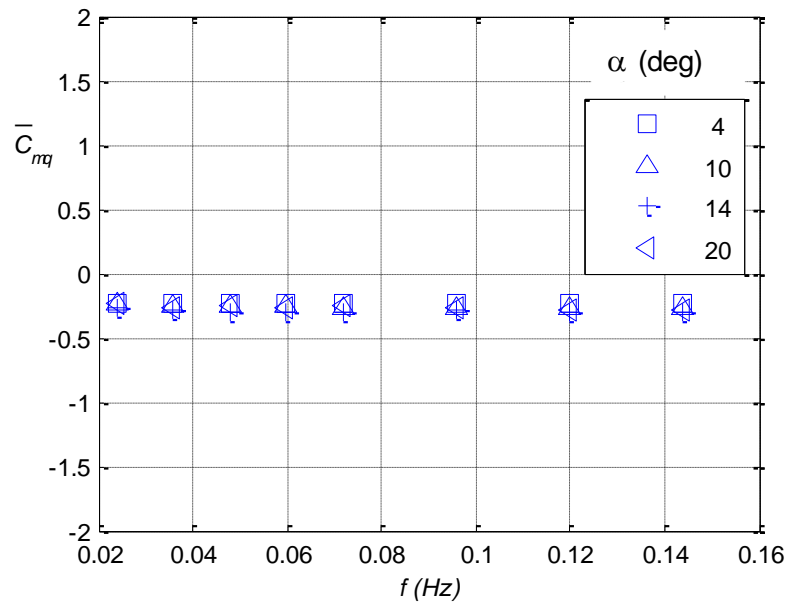

Figure 10. Out-of-phase pitching moment coefficient, $\bar{C}_{m_{q}}, \alpha_{A}=10^{\circ}$.

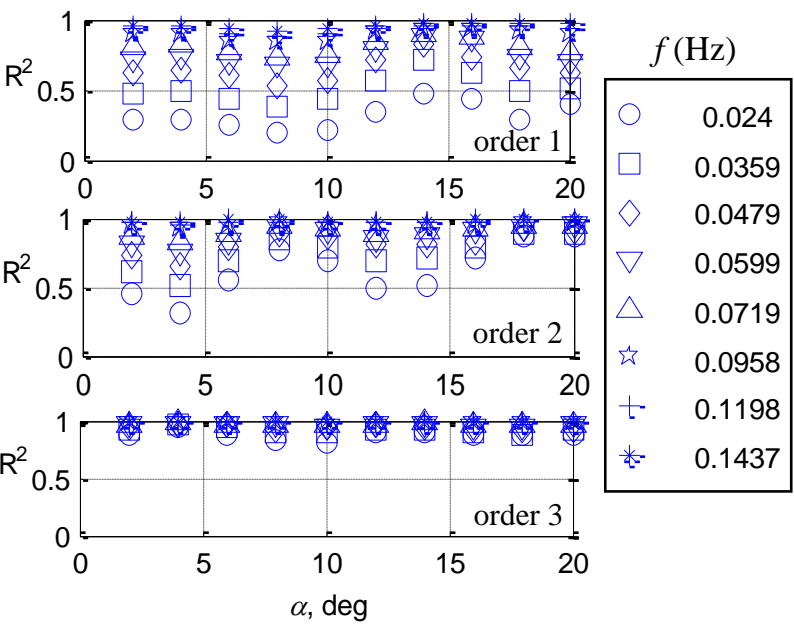

Figure 11. $R^{2}$ for $1^{\text {st }}, 2^{\text {nd }}$, and $3^{\text {rd }}$ order models of $\bar{C}_{m_{q}}, \alpha_{A}=10^{\circ}$.

11

American Institute of Aeronautics and Astronautics 

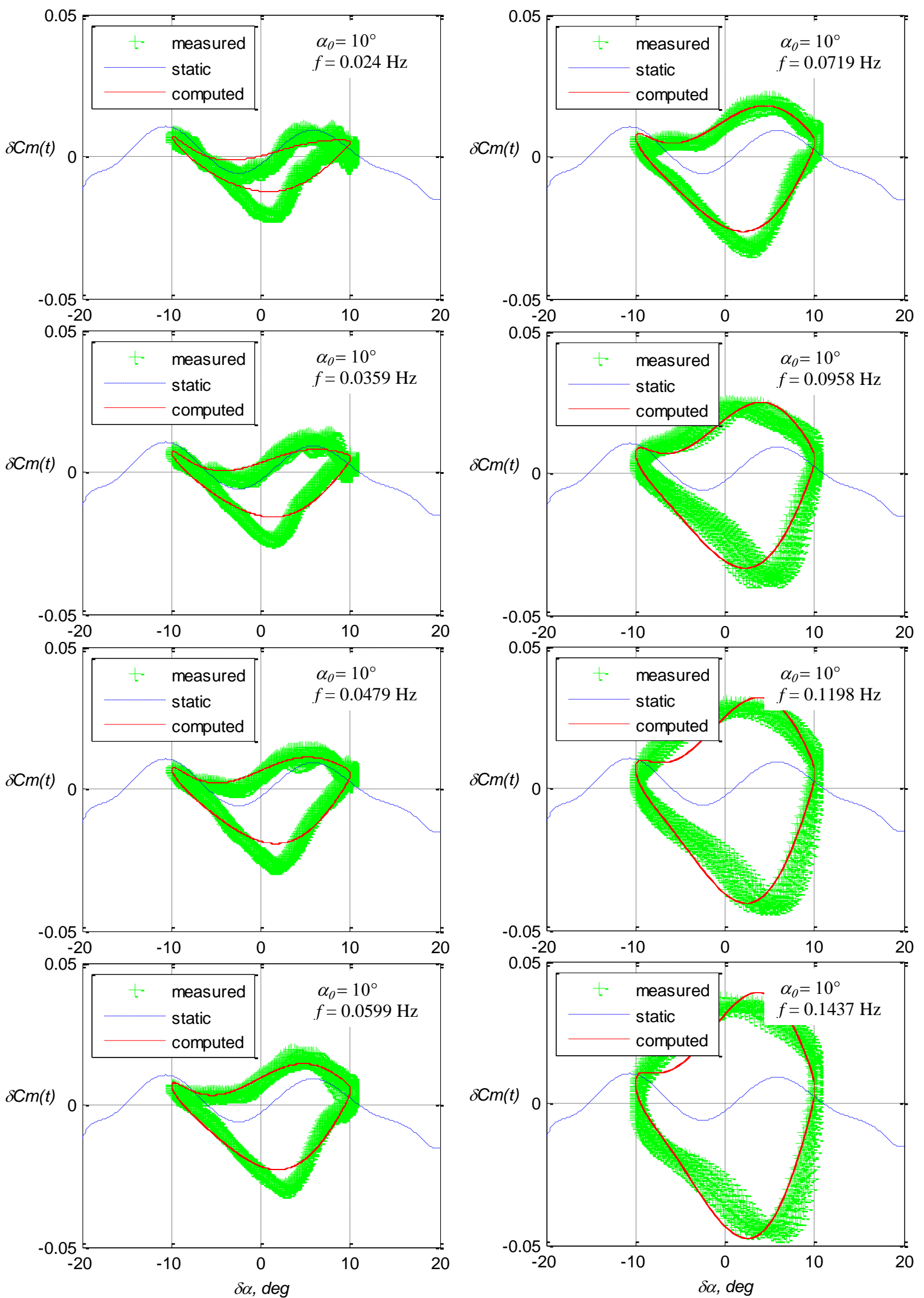

Figure 12. Measured and computed pitching-moment responses at each test frequency, $\alpha_{A}=10^{\circ}$. 12

American Institute of Aeronautics and Astronautics 


\section{Concluding Remarks}

Selected experimental results of a water tunnel study, at Rolling Hills Research Corporation, of the $0.7 \%$ scale NASA HWB-N2A configuration were presented in this paper. Static and dynamic tests about all three axes and flow visualization tests were performed using the HWB model. This work provides new dynamic data to support investigations at NASA Langley in developing advanced transport concepts and to support development of transport aircraft simulations in nonlinear or adverse flight regimes.

Dynamic testing was performed using both single frequency and wide-band motions. Measured data were obtained over an extensive range of angles-of-attack, amplitudes, and frequencies. In this paper analysis was presented for single frequency forced oscillations in the longitudinal axis. The analysis found that HWB does not present significant unsteady behaviors but does show nonlinear characteristics for pitch damping and the static coefficients in the longitudinal axis. Cubic polynomials were required to model the steady flow damping term under large amplitude $\left(10^{\circ}\right)$ forced oscillations in pitch.

\section{References}

${ }^{1}$ Gatlin, Gregory M., Vicroy, Dan D., Carter, Melissa B., "Experimental Investigation of the Low-Speed Aerodynamic Characteristics of a 5.8-Percent Scale Hybrid Wing Body Configuration," $30^{\text {th }}$ AIAA Applied Aerodynamics Conference, AIAA 2012-2669.

${ }^{2}$ Collier, F., Thomas, R., Burley, C., Nickol, C., Lee, M., and Tong, M., "Real Solutions For Environmental Challenges Facing Aviation," ICAS-2010-1.6.1.

${ }^{3}$ Belcastro, Christine M. and Jacobson, Steven R., "Future Integrated Systems Concept for Preventing Aircraft Loss-ofControl Accidents," AIAA Guidance, Navigation, and Control Conference, AIAA 2010-8142.

${ }^{4}$ Belcastro, Christine M., "Validation of Safety-Critical Systems for Aircraft Loss-of-Control Prevention and Recovery," AIAA Guidance, Navigation, and Control Conference, AIAA 2012-4987.

${ }^{5}$ Tobak, Murray, "On the Use of Indicial Function Concept in the Analysis of Unsteady Motions of Wings and Wing-Tail Combinations, NACA Rep. 1188, 1954.

${ }^{6}$ Klein, Vladislav, "Modeling of Longitudinal Unsteady Aerodynamics of a Wing-Tail Combination," NASA CR-1999209547, September, 1999.

${ }^{7}$ Klein, Vladislav and Norderer, Keith D., "Modeling of Aircraft Unsteady Aerodynamic Characteristics. Part I - Postulated Models," NASA TM 109120, May, 1994.

${ }^{8}$ Murphy, Patrick C. and Klein, Vladislav, "Transport Aircraft System Identification from Wind Tunnel Data," AIAA Atmospheric Flight Mechanics Conference, AIAA 2008-6202.

${ }^{9}$ Klein, Vladislav and Murphy, Patrick C., "Estimation of Aircraft Nonlinear Unsteady Parameters From Wind Tunnel Data," NASA TM-1998-208969, December, 1998.

${ }^{10}$ Murphy, Patrick C. and Klein, Vladislav, "Validation of Methodology for Estimating Aircraft Unsteady Aerodynamic Parameters From Dynamic Wind Tunnel Tests," AIAA Atmospheric Flight Mechanics Conference, AIAA 2003-5397, 2003.

${ }^{11}$ Klein, Vladislav, Murphy, Patrick C., and Szyba, Nathan M., "Analysis of Wind Tunnel Oscillatory Data of the F-16XL Aircraft," NASA TM-2004-213246, August, 2004.

${ }^{12}$ Murphy, Patrick C. and Klein, Vladislav, "Transport Aircraft System Identification Using Roll and Yaw Oscillatory Wind Tunnel Data," AIAA Atmospheric Flight Mechanics Conference, AIAA 2010-8122.

${ }^{13}$ Murphy, Patrick C., Klein, Vladislav, Frink, Neal T., and Vicroy, Dan D., "System Identification Applied to Dynamic CFD Simulation and Wind Tunnel Data," AIAA Atmospheric Flight Mechanics Conference, AIAA 2011-6522.

${ }^{14}$ Murphy, Patrick C., Klein, Vladislav, and Frink, Neal T., "Unsteady Aerodynamic Modeling in Roll for the NASA Generic Transport Model," AIAA Atmospheric Flight Mechanics Conference, AIAA 2012-4652.

${ }^{15}$ Murphy, Patrick C. and Klein, Vladislav, "Progressive Aerodynamic Model Identification from Dynamic Water Tunnel Test of the F-16XL Aircraft," AIAA Atmospheric Flight Mechanics Conference, AIAA 2004-5277, August 2004.

${ }^{16}$ Kramer, Brian, and Kerho, Michael, "Aerodynamic Modeling in Unsteady Flight Regimes," SBIR Phase III Final Report, Contract Number NNL12AA36C with NASA Langley Research Center, December 2, 2013.

${ }^{17}$ Vicroy, Dan D., Gatlin, Gregory M., Jenkins, Luther, Murphy, Patrick C., "Low-speed Aerodynamic Investigations of a Hybrid Wing Body Configuration," $32^{\text {th }}$ AIAA Applied Aerodynamics Conference, (submitted for publication).

${ }^{18}$ Murphy, Patrick C. and Klein, Vladislav, "Estimation of Unsteady Aerodynamic Models from Dynamic Wind Tunnel Data," NATO, RTO-MP-AVT-189, 2011.

${ }^{19}$ Klein, Vladislav and Morelli, Eugene, "Aircraft System Identification: Theory and Practice," 1st edition, AIAA Inc., Reston, Virginia, 2006. 